\title{
Modulation of Effective Refractory Period at the Infarct Border-Zone Provides a Mechanism for Focal Arrhythmogenesis
}

\author{
Adam Connolly ${ }^{1}$, Pawel Gawenda ${ }^{1}$, Gernot Plank ${ }^{2}$ and Martin J. Bishop ${ }^{1}$ \\ ${ }^{1}$ King's College London, London, UK; ${ }^{2}$ Medical University of Graz, Austria
}

\begin{abstract}
Border-zone (BZ) tissue, representing viable yet remodelled myocardium surrounding infarct scars, has been strongly correlated with arrhythmogenic risk postmyocardial infarction. How the electrophysiological remodelling in BZ tissue facilitates arrhythmogenesis, particularly of a focal origin, is currently unknown. In this study, we used computational models of human ventricular tissue to quantify spatial changes in action potential duration (APD) and effective refractory period (ERP) in the presence of electrophysiological $B Z$ remodelling. Reductions in sodium channel conductivity to $35 \%$ increased ERP by $>30 \mathrm{~ms}$ relative to healthy tissue in absence of significant changes in APD due to a decrease in tissue excitability. When combined with remodelling of repolarising potassium currents, larger changes in ERP of $>60 \mathrm{~ms}$ occurred, due to concurrent increases in APD. Spatial plots of ERP along interfaces between healthy and $B Z$ regions showed high spatial ERP gradients. Such heterogeneity may facilitate unidirectional block of nearby focal ectopic beats, providing an important focal arrhythmogenenic substrate.
\end{abstract}

\section{Introduction}

Regions of infarct scar tissue, occurring post myocardial infarction, have been strongly correlated with increased risk of reentrant ventricular arrhythmias. Borderzone (BZ) tissue, constituting viable yet remodelled myocardium surrounding infarct scars, is often interspersed with collageneous fibrosis that can effectively slow electrical activation [4]. Although the manner in which the slowed conduction through BZ tissue facilitates the scar's ability to sustain a reentrant circuit is reasonably well understood [2], its potential role in the initial genesis of these reentrant arrhythmias is far less well known.

The electrophysiological properties of $\mathrm{BZ}$ tissue is known to be significantly different to the neighbouring healthy myocardium, and may underlie its role as an arrhythmogenic substrate. Isolated myocytes from BZ tis- sue have been shown to display significant remodelling corresponding to the down-regulation of numerous individual ion channels and transport processes [3]. Downregulation of the fast sodium current $\left(I_{N a}\right)$ and the L-type calcium current $I_{C a L}$ cause reduced excitability, lower action potential amplitude, decreased upstroke velocity and consequently slow conduction. A reduction in repolarising potassium currents $\left(I_{K r}, I_{K s}, I_{t o}\right)$ causes prolongation of the action potential, and can give rise to the formation of early-afterdepolarisation. As well as remodelling of ionic currents, cell-to-cell conductivity is often affected by a combination of down-regulation and re-distribution of Cx-43, in addition to physical separation of myocytes by interstitial fibrotic clefts as well as the potential presence of electrically-coupled myofibroblasts [4].

We have recently demonstrated $[5,6]$ that changes in local electrotonic loading (due to the presence of tissue boundaries, small cavities and rapid tissue expansions), can significantly affect the excitability of cardiac tissue, causing a corresponding spatial variation in local effective refractory period (ERP). Such spatial gradients in ERP were witnessed in absence of any significant changes in action potential duration (APD), and were shown to be responsible for causing uni-directional block and subsequent arrhythmia induction. In this study, we aim to investigate whether the reduction in excitability within BZ tissue due to ionic remodelling may combine with the known increases in APD in these regions, to introduce significant gradients in ERP that may facilitate uni-directional block at the boundary between BZ and healthy myocardium.

\section{Methods}

The computational domain used to perform simulations constituted a square of tissue of size $20 \mathrm{~mm}$, discretised into a structured quadrilateral finite element mesh, with nodal spacing $100 \mu \mathrm{m}$. The tissue was divided in half along the $y$-axis to represent a heterogeneous region of tissue in which the healthy myocardium adjoins the BZ tissue. The right-hand side was assigned BZ ionic properties whilst the left-hand side was assigned to be healthy myocardium, shown in the schematic Figure 1. Pacing 


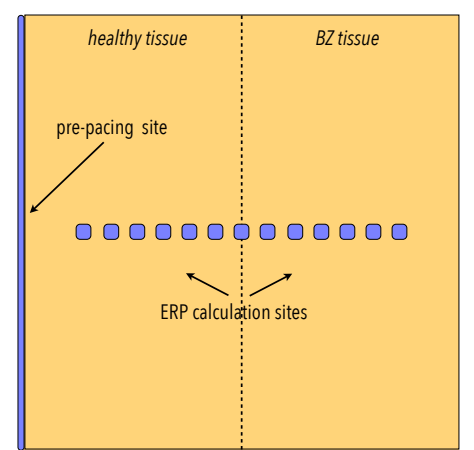

Figure 1. Diagram of tissue setup showing pacing stimulus site (blue bar) along with sites of ERP calculation.

stimuli were applied to the left-hand edge of the tissue ( $y=-10 \mathrm{~mm}$ edge). In all simulations, the cell models were pre-paced for 50 beats at a cycle length of $500 \mathrm{~ms}$ and state variables saved, prior to use within the tissue models. The tissue was subsequently pre-paced at this location for 10 beats. APD was computed for the 10th beat.

ERP was computed following the 10th beat at a series of different spatial locations along the central $x$-axis between $x=-7 \mathrm{~mm}$ and $x=+7 \mathrm{~mm}$, using a stimulus region of size $200 \times 200 \mu \mathrm{m}$ (Figure 1). The protocol was also repeated using pre-pacing applied to the right-hand edge of the tissue $(y=+10 \mathrm{~mm}$ edge $)$, as the heterogeneous distribution of ionic conductances is expected to interact with differing gradients in repolarisation in these two scenarios.

Electrophysiological activity was computed using a finite element monodomain representation with the Cardiac Arrhythmia Research Package [7]. Ionic dynamics were represented by the ten Tusscher human ventricular cell model. Modifications to BZ tissue were based-on those used previously in the literature [8], involving reductions in ionic conductances of: $G_{K r}$ to $30 \%, G_{K s}$ to $20 \%, G_{C a L}$ to $30 \%$, and $G_{N a}$ to $35 \%$ of their respective normal values. An alternative tissue setup was also considered in which the $\mathrm{BZ}$ only had a reduction in $G_{N a}$ to $35 \%$ imposed, with all other conductances being kept at their healthy values. Tissue conductivity of healthy myocardium was assigned an isotropic value of $0.2 \mathrm{~S} / \mathrm{m}$, while the conductivity of BZ tissue was further reduced to $0.05 \mathrm{~S} / \mathrm{m}$.

\section{Results}

\subsection{Effect of Reduced Sodium on APD \& ERP across Infarct BZ}

Initially, we examine the effects of the tissue setup in which $G_{N a}$ is reduced to $35 \%$ in the $\mathrm{BZ}$ tissue, but all other ionic conductances are initially kept at their default healthy values. We also consider two different cases of tissue conductivity with the $\mathrm{BZ}$ tissue either assigned the lower conductivity of $0.05 \mathrm{~S} / \mathrm{m}$ or alternatively the same conductivity as the healthy regions of $0.2 \mathrm{~S} / \mathrm{m}$.

Figure 2A plots the spatial variation in APD along the horizontal mid-line of the tissue (Figure 1), whilst Figure $2 \mathrm{~B}$ plots the spatial variation in ERP along the same line, calculated as described above, for a BZ conductivity of $0.2 \mathrm{~S} / \mathrm{m}$. Figure $2 \mathrm{C} \& \mathrm{D}$ show similar APD and ERP data for the case of a BZ conductivity of $0.05 \mathrm{~S} / \mathrm{m}$. Two different plots are shown in each panel for the cases of leftto-right or right-to-left wavefront propagation.

In the case of APD, little spatial variation is seen for either BZ conductivity. Here, any effect of a slightly increased APD in the BZ where $G_{N a}$ is reduced interacts with the well-known reduction in APD along the direction of propagation due to electrotonic interactions, meaning slight differences are seen between left-to-right and rightto-left propagation. Despite little differences in APD, substantial differences in ERP are witnessed between regions. In Figure 2B, ERP is seen to differ by approximately $40 \mathrm{~ms}$ between healthy and $\mathrm{BZ}$ regions, with a very rapid change between the two, leading to a correspondingly high ERP spatial gradient around the centre line. In Figure 2D, a similarly rapid change in ERP is seen around the centre of the tissue, increasing by approximately $40-60 \mathrm{~ms}$, again giving a high gradient in ERP in this area. However, ERP decreases again deeper into the $\mathrm{BZ}$ tissue, falling to a value approximately $20 \mathrm{~ms}$ greater than deep within the healthy tissue (comparing the far left- and right-side of the tissue).

This difference between ERP in the BZ for different BZ tissue conductivity is due to the fact that a lower conductivity reduces the electrotonic loading at the stimulus site, facilitating focal capture and lowering ERP compared to higher conductivity regions. However, this acts against the raised APD and reduced excitability in the BZ, both of which act to raise ERP. A high ERP is seen in the centre of the domain as here the tissue on the left side is still highly conducting, increasing the electronic loading. Thus, the overall loading on $\mathrm{BZ}$ in the centre of the domain is larger here than deep within the BZ (further to the right).

\subsection{Combined Effect of Other Ionic Cur- rent Modulations}

Figure 3 shows similar data to Figure 2, but in this case the BZ tissue has reductions in other ionic conductances imposed, in addition to reductions in $G_{N a}$. Contrary to the previous scenario in Figure 2, in this case, a significant variation in APD is witnessed between BZ and healthy myocardium, with a smooth transition in between (smoothed by electronic interactions), which is seen for both reduced 

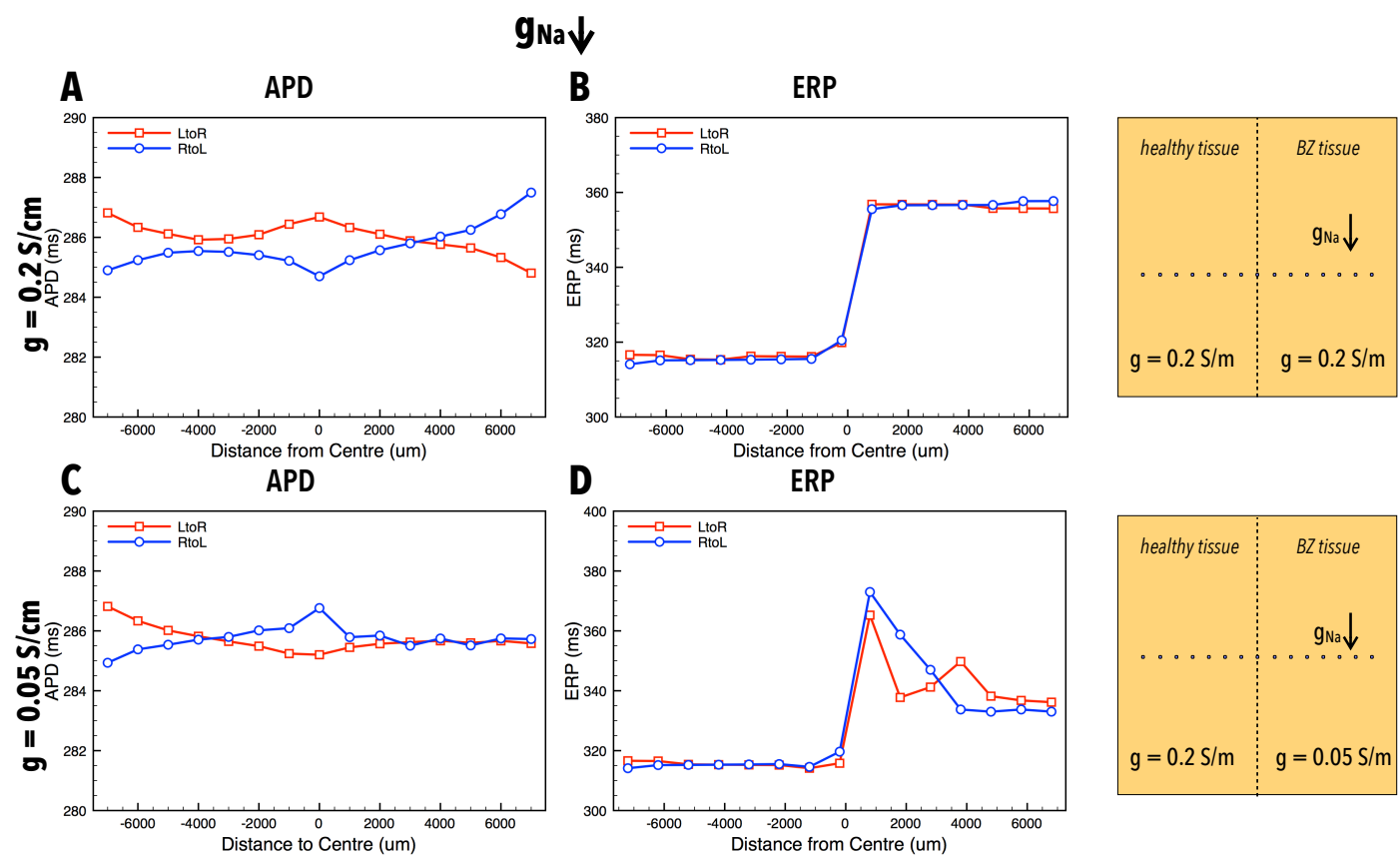

Figure 2. Healthy myocardial tissue (left) proximal to BZ tissue (right) separated by vertical black dotted-line, plotting APD and ERP along midway horizontal lines across the tissue (row of blue squares). Plots are shown corresponding to measurements taken following both right-to-left (RtoL) and left-to-right (LtoR) pre-pacing across the tissue. Only gNa is reduced by a factor of 0.35 in BZ tissue. BZ tissue conductivity is $0.2 \mathrm{~S} / \mathrm{m}$ (upper panels) and $0.05 \mathrm{~S} / \mathrm{m}$ (lower panels).
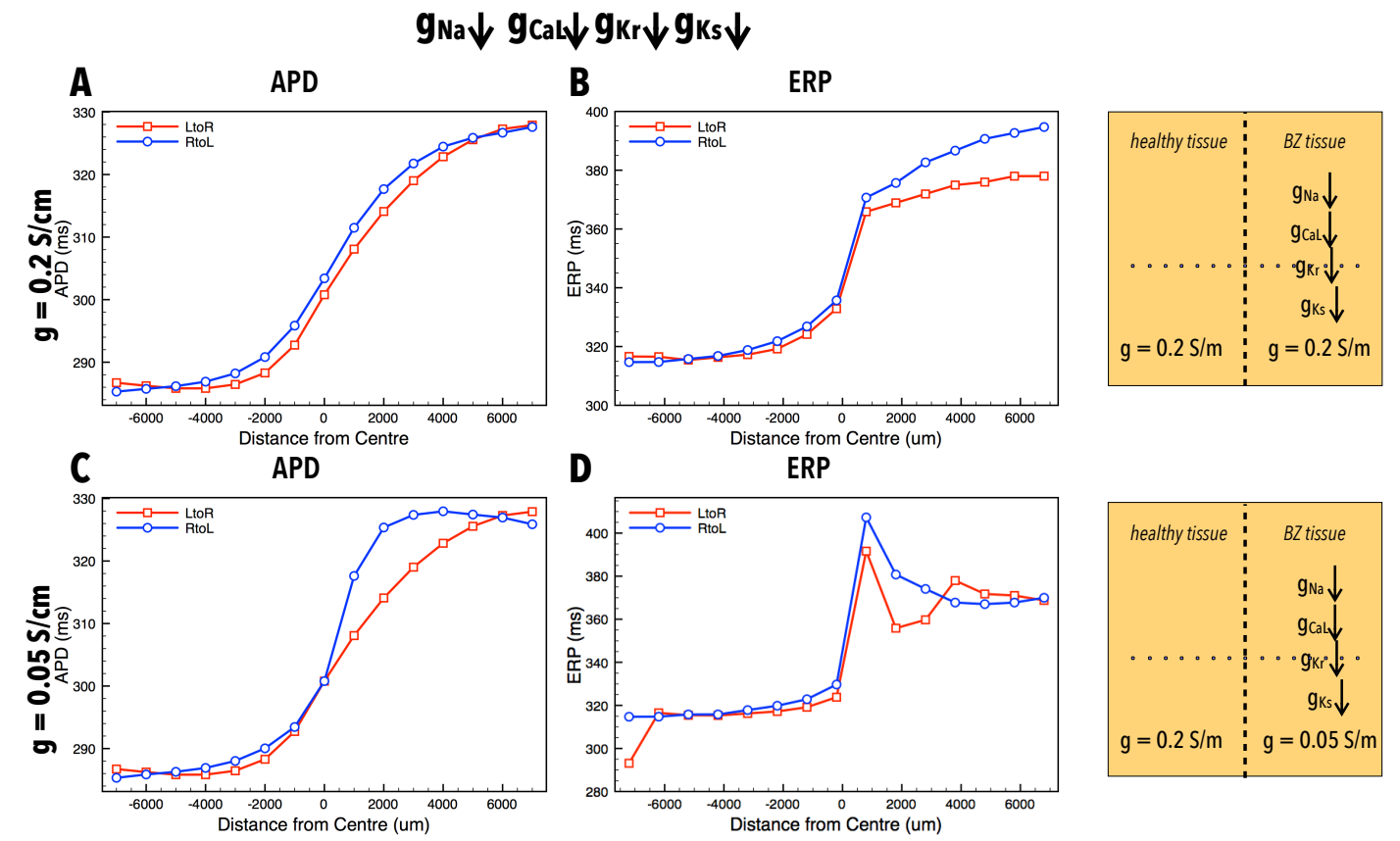

Figure 3. Healthy myocardial tissue (left) proximal to BZ tissue (right) separated by vertical black dotted-line, plotting APD and ERP along midway horizontal lines across the tissue (a row of blue squares). Plots are shown corresponding to measurements taken following both right-to-left (RtoL) and left-to-right (LtoR) pre-pacing across the tissue. Full EP remodelling of all relevant currents is made in BZ tissue. BZ tissue conductivity is $0.2 \mathrm{~S} / \mathrm{m}$ (upper panels) and $0.05 \mathrm{~S} / \mathrm{m}$ (lower panels). 
(panel A) and normal (panel C) conductivity. Such an elevated APD within the BZ is due to the now imposed reduction in conductivities of potassium currents responsible for the repolarisation phase of the action potential.

Figures $3 \mathrm{~B} \& \mathrm{D}$ show that the increase in APD between normal tissue and BZ in panels A \& $\mathrm{C}$ acts to augment the rise in ERP seen previously in Figure 2 in the case where only $G_{N a}$ was modulated (with little change in APD seen). In the case of similar tissue conductivity in the healthy and $\mathrm{BZ}$ regions (panel B), a significant increase in ERP is seen, rising by up to almost $80 \mathrm{~ms}$ between healthy and BZ tissue. In the case where BZ tissue conductivity is reduced (panel D), the initial rise in ERP is similar to panel $B$ in the tissue transition region, however, further into the BZ tissue, ERP reduces due to the reduced electrotonic loading due to the lower tissue conductivity.

\section{Discussion \& Conclusions}

BZ tissue surrounding regions of infarct scar is known to be a signature of elevated risk of reentrant arrhythmias. However, the mechanisms by which BZ tissue may initiate of reentry is not well understood. In this work, we have demonstrated how electrophysiological remodelling of ionic conductances within BZ can lead to significant gradients in ERP between BZ and healthy myocardium, which may provide an important mechanism of arrhythmia initiation via uni-directional block.

ERP measures how soon tissue can be excited following an initial pacing stimulus. Here, we have shown that the decrease in sodium channel conductance $G_{N a}$ alone can lead to significant increases in ERP within BZ tissue, due to the related reduction in excitability (without any change in APD). However, when combined with concurrent changes in other ionic currents frequently measured experimentally in BZ tissue (primarily in repolarising potassium currents), the corresponding increase in APD in BZ tissue acts alongside this reduction in excitability due to reduced $G_{N a}$ to further raise ERP in BZ regions.

We have further shown that such an increase in ERP in BZ tissue can lead to a high spatial ERP gradient between healthy myocardium and the BZ region. Such gradients are present both when the initial wavefront (prior to ERP calculation) occurs from healthy-to-BZ as well as from BZto-healthy tissue. In our previous studies $[5,6]$, we have demonstrated how gradients in ERP (caused in these cases due to differences in electrotonic loading brought about by structural heterogeneity) can facilitate uni-directional conduction block following a focal ectopic beat applied close to the ERP of tissue experiencing a high gradient. We suggest that a similar mechanism may occur in this scenario. As BZ tissue is known to be more susceptible to afterpolarisations [3], such a mechanism of conduction block due to ERP gradients, driven by local electrophysiological re- modelling, could help explain increased arrhythmogenic risk of BZ tissue.

\section{Acknowledgements}

The authors acknowledge the British Heart Foundation under Project Grant number PG/14/66/30927.

\section{References}

[1] Rutherford SL, Trew ML, Sands GB, LeGrice IJ, Smaill BH. High-Resolution 3-Dimensional Reconstruction of the Infarct Border Zone: Impact of Structural Remodeling on Electrical Activation. Circ Res 2012;111:301-11.

[2] Stevenson WG. Ventricular tachycardia after myocardial infarction: from arrhythmia surgery to catheter ablation. Journal of Cardiovascular Electrophysiology 1995;6(10):942950.

[3] Nattel S, Maguy A, Le Bouter S, Yeh YH. Arrhythmogenic Ion-Channel Remodeling in the Heart: Heart Failure, Myocardial Infarction, and Atrial Fibrillation. Physiol Rev April 2007;87:425-56.

[4] de Jong S, van Veen TAB, van Rijen HVM, de Bakker JMT. Fibrosis and cardiac arrhythmias. J Cardiovasc Pharm 2011; 57:630-8.

[5] Bishop MJ, Connolly A, Plank G. Structural Heterogeneity Modulates Effective Refractory Period: A Mechanism of Focal Arrhythmia Initiation. PLoS ONE 2014;9:e109754.

[6] Connolly A, Trew M, Smaill B, Plank G, Bishop M. Local Gradients in Electrotonic Loading Modulate the Local Effective Refractory Period: Implications. IEEE Trans Biomed Eng 2015;

[7] Vigmond E, Hughes M, Plank G, Leon LJ. Computational tools for modeling electrical activity in cardiac tissue. J Electrocardiol 2003;36:69-74.

[8] Arevalo H, Plank G, Helm P, Halperin H, Trayanova N. Tachycardia in Post-Infarction Hearts: Insights from 3D Image-Based Ventricular Models. PLoS ONE July 2013; 8(7):e68872.

[9] Ringenberg J, Makarand D, Filgueiras-rama D, Pizarro G, Ibanez B, Peinado R, Merino J, Berenfeld O, Devabhaktuni V. Effects of Fibrosis Morphology on Reentrant Ventricular Tachycardia Inducibility and Simulation Fidelity in Patient-Derived Models. Clinical Medicine Insights Cardiology 2014;

Address for correspondence:

Dr Martin J. Bishop Department of Biomedical Engineering, King's College London, St Thomas' Hospital, London SE1 7EH, UK

martin.bishop@kcl.ac.uk 